\title{
Shortness of breath while lying down: a woman with orthopneic asthma
}

\author{
Chou-Han Lin, Mong-Wei Lin, Jin-Shing Chen, Chong-Jen Yu
}

See related practice article by Lee and Cheng, page 80

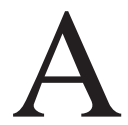

36-year-old woman with a six-month history of worsening shortness of breath was admitted to hospital for investigation. She had received a diagnosis of bronchial asthma six months earlier, for which treatment with steroids and bronchodilators had been ineffective. Her history included allergic rhinitis and an eight pack-year history of smoking, which she had stopped since her diagnosis of asthma.

On admission, the patient was in mild respiratory distress. She had a temperature of $36.5^{\circ} \mathrm{C}$, a pulse rate of 90 beats $/ \mathrm{min}$, a respiratory rate of 22 breaths/min and a blood pressure of 116/64 mm Hg. She had diffuse wheezing on expiration and during the first half of inspiration. We noticed that she had orthopnea and had to use three pillows for relief. She had not mentioned this symptom to her treating physicians. Her distress when lying down occurred even though her oxygen saturation remained above $98 \%$ on $2 \mathrm{~L}$ of oxygen per minute via nasal cannula. A pulmonary function test showed a forced expiratory volume in the first

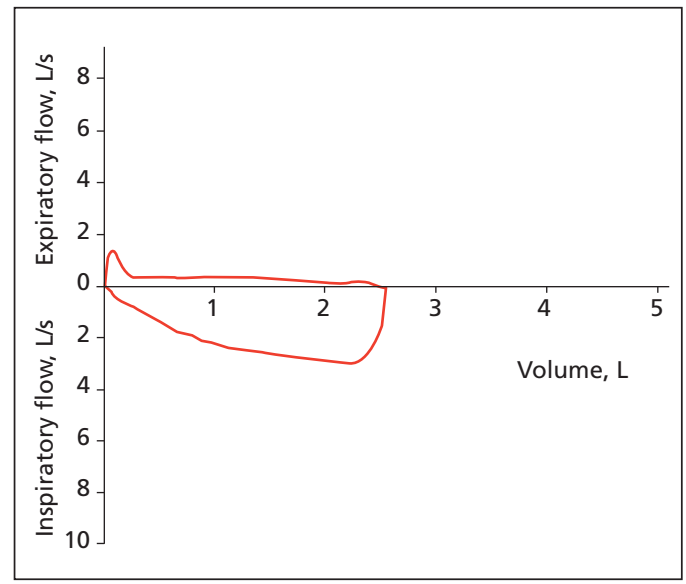

Figure 1: Flow-volume loop from a 36-year-old woman with orthopnea, showing a plateau phase on the expiratory arm. second of expiration $\left(\mathrm{FEV}_{1}\right)$ of $17.5 \%$ of predicted, a forced vital capacity (FVC) of $75.7 \%$ and an $\mathrm{FEV}_{1} / \mathrm{FVC}$ ratio of $20.4 \%$ of predicted. Her flow-volume loop showed slowing and flattening in the expiratory limb (Figure 1), which led us to re-examine her chest radiograph taken on admission (Figure 2). The tracheal air column was not deviated, and no conspicuous lesions were noted.

\section{What is your diagnosis?}

a. Upper airway obstruction

b. Left-sided heart failure (cardiac asthma)

c. Status asthmaticus

d. Hepatopulmonary syndrome

e. Exacerbation of chronic obstructive pulmonary disease

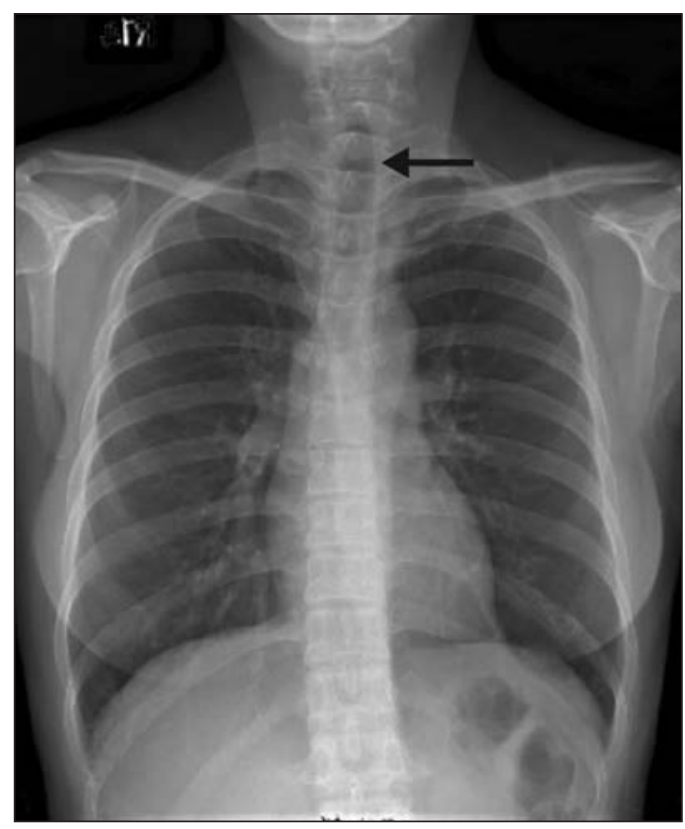

Figure 2: Posteroanterior chest radiograph, showing a straight tracheal air column (arrow) without narrowing or deviation.
Competing interests: None declared.

This article has been peer reviewed.

Correspondence to: Dr. Chong-Jen Yu, jefferycjyu@ntu.edu.tw

CMAJ 2011. DOI:10.1503 /cmaj.081801 


\section{What is your next step?}

a. Give magnesium sulfate along with standard combination of oxygen, bronchodilators and steroids

b. Give antibiotics along with standard combination of oxygen, bronchodilators and steroids

c. Order a lateral chest radiograph and a computed tomography (CT) scan of the chest

d. Arrange contrast echocardiography

e. Administer intravenous nitroglycerin with an angiotensin-converting-enzyme inhibitor

The diagnosis is (a) upper airway obstruction, and the next step is (c) order a lateral chest radiograph and a CT scan. We made the diagnosis with the help of the flow-volume loop, which was consistent with intrathoracic upper airway obstruction. Her lateral chest radiograph showed a tumour-like opacity in the trachea (Figure 3). The CT scan showed a tracheal tumour with almost total obstruction of the tracheal lumen (Figure 4). Bronchoscopy showed a whitish bosselated tumour arising from the anterior wall of the trachea $6 \mathrm{~cm}$ below the vocal cords.

The patient underwent segmental resection of the trachea with anastomosis. A whitish elastic tumour $(2 \times 2 \times 2 \mathrm{~cm})$ was found $3 \mathrm{~cm}$ above the carina, extending from the anterior tracheal wall (Figure 5). Histopathology results were consistent with a pleomorphic adenoma. After resection, her wheezing and orthopnea disappeared.

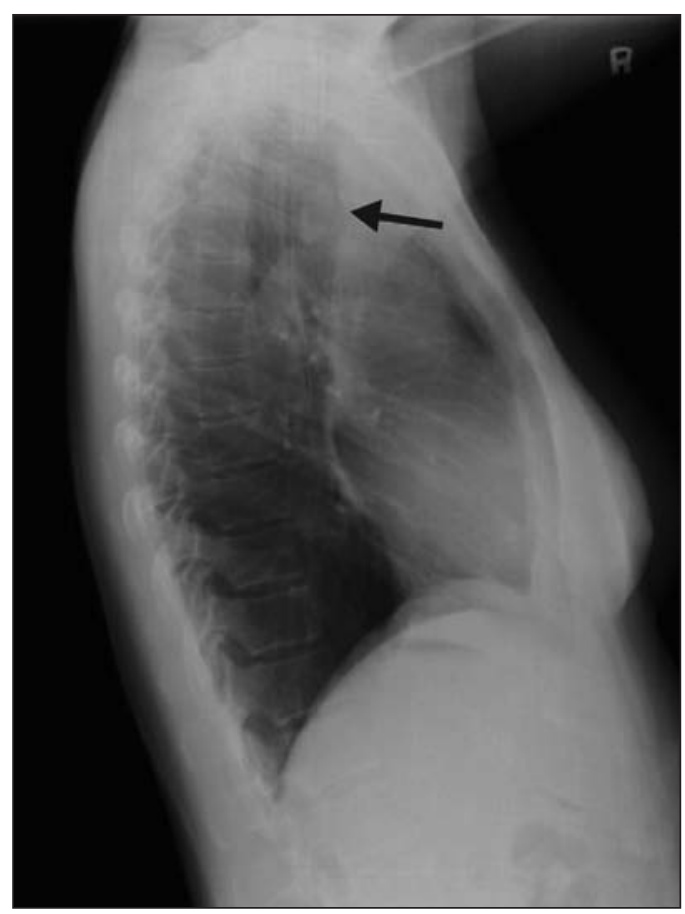

Figure 3: Lateral chest radiograph, showing an opacity (arrow) arising from the anterior tracheal wall with a patent posterior tracheal lumen.

\section{Discussion}

Orthopnea is often associated with congestive heart failure, chronic obstructive pulmonary disease, anterior mediastinal tumours and diaphragmatic weakness. Tracheal tumours, as in our patient, are a rare cause of orthopnea. ${ }^{1}$ Because our patient's tumour stalk emerged from the anterior tracheal wall, part of the tumour approached the opposing membranous portion and occluded the lumen when she lay down (Figure 4).

The posteroanterior chest radiograph is an appropriate initial imaging study for assessing dyspnea. However, it is a relatively poor tool for identifying tumours situated distally in the anterior or posterior tracheal wall. A lateral chest radiograph is helpful for assessing tracheal tumours in these locations. Most pleomorphic adenomas are located in the upper third of the trachea. ${ }^{2}$ However, the intrathoracic location of our patient's tumour lowered its visibility on the posteroanterior view of the chest radiograph. A lateral view provides better exposure, without interference from the thoracic spine and sternum. ${ }^{3}$

Computed tomography is useful in detecting and assessing tracheal tumours, given that not all soft-tissue lesions are visible on chest radiography. As in our case, pulmonary function testing can be helpful in diagnosing tracheal lesions, with confirmation provided by direct visualization using bronchoscopy.

\section{Differential diagnosis}

Orthopnea occurs most frequently in patients with heart failure. These patients find relief in an upright position because this posture reduces the demand on their pulmonary circulation. A constellation of clinical symptoms and signs such as paroxysmal nocturnal dyspnea, distention of the veins in the neck and lung crackles can also be found in patients with heart failure. The subacute course and lack of these features in our patient ruled out this disease as a cause of her orthopnea.

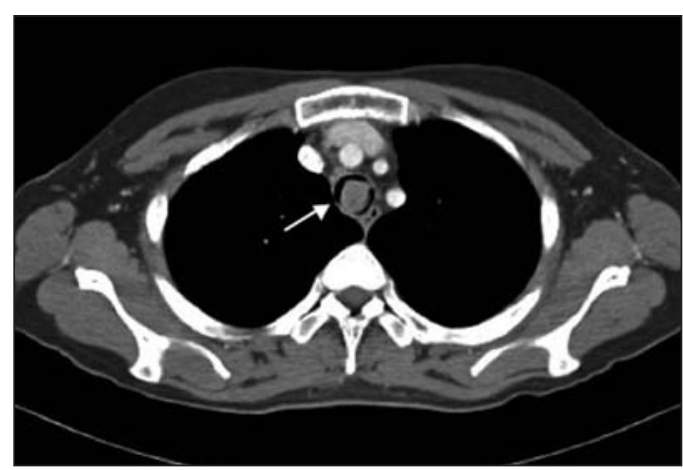

Figure 4: Chest computed tomography scan, showing a pedunculated tumour (arrow) nearly obliterating the tracheal lumen. 


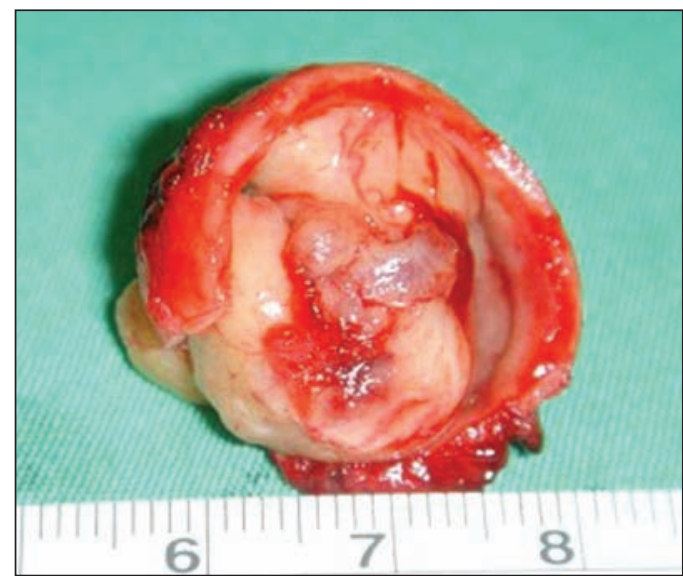

Figure 5: Tracheal tumour attached to the anterior of the tracheal ring.

Also, her chest radiograph did not show cardiomegaly or pulmonary edema, which are often associated with heart failure.

Hepatopulmonary syndrome develops in the setting of chronic liver disease. The resulting dilated pulmonary capillaries create intrapulmonary shunts. Patients with this condition often exhibit platypnea (shortness of breath relieved with lying down) or orthodeoxia (deoxygenation in the sitting or standing position) rather than orthopnea.

Occasionally, orthopnea is also found in patients with chronic obstructive lung disease and asthma, since the upright position may be associated with reduced pooling of lung secretions and improved diaphragmatic excursion. Orthopnea tends to be a late manifestation of chronic lung disease. Wheezing is commonly observed in chronic obstructive pulmonary disease and asthma, and subacute or chronic upper airway obstruction can mimic diseases of peripheral airflow obstruction. Our patient's wheezing pattern, however, which extended from expiration to inspiration, would be unusual in these conditions.

\section{Pulmonary function testing}

Not only is spirometry a diagnostic tool for chronic obstructive lung disease and asthma, it can also help identify upper airway obstruction (Figure 6). ${ }^{4} \mathrm{FEV}_{1}$, the spirometric parameter used for assessing chronic obstructive pulmonary disease and asthma, is less sensitive in

\begin{tabular}{lcccccc|}
\hline & & & & & \\
& Small airway obstruction & \multicolumn{3}{c|}{ Upper airway obstruction } \\
\hline & COPD & Asthma attack & $\begin{array}{c}\text { Variable } \\
\text { intrathoracic }\end{array}$ & $\begin{array}{c}\text { Variable } \\
\text { extrathoracic }\end{array}$ & Fixed \\
\hline FVC & $\mathrm{N}$ to $\downarrow$ & $\downarrow$ & $\mathrm{N}$ & $\mathrm{N}$ & $\mathrm{N}$ \\
\hline FEV 1 & $\downarrow$ & $\downarrow$ & $\downarrow$ & $\mathrm{N}$ & $\downarrow$ \\
\hline FEV $/ \mathrm{FVC}$ & $\downarrow$ & $\mathrm{N}$ to $\downarrow$ & $\downarrow$ & & $\mathrm{N}$ & $\downarrow$ \\
\hline $\begin{array}{l}\text { Flow-volume } \\
\text { loop }\end{array}$ & & & & & & \\
\hline
\end{tabular}

Figure 6: Typical findings of pulmonary function tests in patients with small (lower) airway and upper airway obstruction. COPD = chronic obstructive pulmonary disease, $F V C$ = forced vital capacity, FEV $_{1}=$ forced expiratory volume in the first second of expiration. Reprinted, with permission, from Hyatt et al. ${ }^{4}$ Copyright @ 2009 Lippincott Williams \& Wilkins (http://lww.com).

diagnosing upper airway obstruction. ${ }^{5}$ For this condition, the ratio of maximal expiratory to maximal inspiratory flow at $50 \%$ of vital capacity $\left(\mathrm{MEF}_{50}: \mathrm{MIF}_{50}\right.$ ratio $)$ is more useful. The $\mathrm{MEF}_{50}: \mathrm{MIF}_{50}$ ratio, normally between 0.9 and 1.0 , will decrease to less than 0.2 in patients with an intrathoracic obstruction or increase to more than 1.0 in those with an extrathoracic obstruction. ${ }^{6}$ As shown in Figure 6, direct inspection of the flow-volume loop is helpful when interpreting spirometric data and can be used along with specific flow calculations to make the diagnosis of upper airway obstruction. ${ }^{6}$

\section{References}

1. Gaissert HA, Grillo HC, Shadmehr B, et al. Uncommon primary tracheal tumors. Ann Thorac Surg 2006;82:268-73.

2. Aribas OK, Kanat F, Avunduk MC. Pleomorphic adenoma of the trachea mimicking bronchial asthma: report of a case. Surg Today 2007;37:493-5.

3. Li W, Ellerbroek NA, Libshitz HI. Primary malignant tumors of the trachea; a radiologic and clinical study. Cancer 1990;66:894-9.

4. Hyatt RE, Scanlon PD, Nakamura M. Interpretation of pulmonary function tests: a practical guide. 3rd ed. Philadelphia: Lippincott Williams \& Wilkins; 2009. Figure 2-7.

5. Miller RD, Hyatt RE. Obstructing lesions of the larynx and trachea: clinical and physiologic characteristics. Mayo Clin Proc 1969:44:145-61.

6. Lunn WW, Sheller JR. Flow volume loops in the evaluation of upper airway obstruction. Otolaryngol Clin North Am 1995;28: 721-9.

Affiliations: From the Division of Chest Medicine, Department of Internal Medicine (C.-H. Lin), Far Eastern Memorial Hospital, Taipei, Taiwan; the Division of Thoracic Surgery, Department of Surgery (M.-W. Lin, Chen), National Taiwan University Hospital, Taipei, Taiwan; and the Division of Chest Medicine, Department of Internal Medicine ( $\mathrm{Yu}$ ),

National Taiwan University Hospital, Taipei, Taiwan 